\title{
Physical and computational aspects of shock waves over power-law leading edges
}

\author{
Wilson F. N. Santos \\ Combustion and Propulsion Laboratory, National Institute for Space Research, \\ Cachoeira Paulista, São Paulo 12630-000, Brazil
}

(Received 10 April 2007; accepted 15 October 2007; published online 15 January 2008)

\begin{abstract}
Computations using the direct simulation Monte Carlo (DSMC) method are presented for hypersonic flow on power-law shaped leading edges. The primary aim of this paper is to examine the geometry effect of such leading edges on the shock-wave structure. The sensitivity of the shock-wave shape, shock-wave thickness, and shock-wave standoff distance to shape variations of such leading edges is investigated by using a model that classifies the molecules in three distinct classes: (1) undisturbed freestream, (2) reflected from the boundary, and (3) scattered, i.e., molecules that had been indirectly affected by the presence of the leading edge. The analysis showed that, for power-law shaped leading edge with exponent between $2 / 3$ and 1 , the shock wave follows the body shape. It was found that, at the vicinity of the nose, the shock-wave power-law exponent is $1 / 2$. Far from the nose, calculations showed that the shock-wave shape is in surprising qualitative agreement with that predicted by the hypersonic small disturbance theory for the flow conditions considered. () 2008 American Institute of Physics. [DOI: 10.1063/1.2831135]
\end{abstract}

\section{INTRODUCTION}

The importance of the lift-to-drag ratio is well known to all hypersonic vehicle designers since it gives the aerodynamic efficiency of the vehicle. With the renewed interest in aerodynamically efficient hypersonic vehicles, high-lift and low-drag configurations, waveriders ${ }^{1}$ have been considered as a promising concept for a high aerodynamic performance. Waverider configuration is a shape designed analytically with infinitely sharp leading edges for shock wave attachment. Because the shock wave is attached to the leading edge of the vehicle, the upper and lower surfaces of the vehicle can be designed separately. Furthermore, this attached shock prevents spillage of higher pressure air from the lower side of the vehicle to the upper side. As a result of this attached shock, waverider has been shown to have the potential to provide high values for the lift-to-drag ratio at high Mach numbers.

Nonetheless, it is known that very sharp leading edge is not practical for a number of reasons: (1) it is difficult to manufacture, (2) some blunting is required for structural strength, and (3) the heat transfer to the nose is severe at high Mach numbers. In this context, any practical waverider will have some degree of bluntness dictated by either manufacturing or heating requirements. Coupled with viscous effects, the resulting shock wave will exhibit a standoff distance. In addition, shock wave detachment will allow pressure leakage from the lower surface of the vehicle to the upper surface, thereby degrading the aerodynamic performance of the vehicle. $^{2}$

Usually, a round leading edge with constant radius of curvature (circular cylinder) near the stagnation point has been chosen for blunting geometry. Nevertheless, shock detachment distance on a cylinder, with associated leakage, scales with the radius of curvature. With this perspective in mind, power-law shaped leading edges $\left(y \propto x^{n}, 0<n<1\right)$ may provide the required bluntness for heat transfer and manufacturing concerns with reduced departures from ideal aerodynamic performance. This concept is based on the work of Mason and Lee, ${ }^{3}$ who have pointed out, based on Newtonian flow analysis, that power-law shapes exhibit both blunt and sharp aerodynamic properties. They suggested the possibility of a difference between shapes that are geometrically sharp and shapes that behave aerodynamically as if they were sharp.

A great deal of experimental and theoretical works ${ }^{4-20}$ has been carried out previously on power-law forms representing blunt geometries. The major interest in these works is twofold: (1) for Refs. 4-7, the interest had gone into finding solutions to the hypersonic small disturbance form of the inviscid adiabatic-flow equations, since the equations of motion for hypersonic flow over slender bodies can be reduced to simpler form by incorporating the hypersonic slenderbody approximations; ${ }^{21}$ (2) for Refs. $8-20$, the interest has gone into considering the power-law shape as possible candidate for blunting geometries of hypersonic leading edges, such as hypersonic waverider vehicles.

Lees and Kubota ${ }^{4}$ observed that similarity exists for hypersonic flows whenever the shock shape follows a powerlaw variation with the streamwise distance, provided the hypersonic slender body equations are considered in the limit as $\left(M_{\infty} \tau\right)^{-2} \rightarrow 0$, where $M_{\infty}$ is the freestream Mach number and $\tau$ is a characteristic shock slope. According to their work, energy considerations combined with a detailed study of the equations of motion show that flow similarity is possible for a class of bodies of the form $x^{n}$, provided that $2 / 3<n<1$ for a two-dimensional body and $1 / 2<n<1$ for an axisymmetric body. The similarity solutions referred herein are solutions for self-similar flows, i.e., flows in which the flowfield between the shock wave and the body can be 
expressed in terms of functions which, in suitable coordinates, are independent of one of the coordinate directions.

Of particular significance on power-law shapes are the works by Santos and Lewis. ${ }^{12-18}$ Through the use of the direct simulation Monte Carlo (DSMC) method, they found that the stagnation point heating behavior for power-law leading edges with finite radius of curvature, $n=1 / 2$, followed that predicted for classical blunt body in that the heating rate is inversely proportional to the square root of curvature radius at the stagnation point. For those power-law leading edges with zero radii of curvature, $n>1 / 2$, it was found that the stagnation point heating is not a function of the curvature radius at the vicinity of the leading edges, but agreed with the classical blunt body behavior predicted by the continuum flow far from the stagnation point. Results were compared to a corresponding circular cylinder to determine which geometry would be better suited as a blunting profile. Their analysis also showed that power-law shapes provided smaller total drag than the circular cylinder, typically used in blunting sharp leading edges for heat transfer considerations. However, circular cylinders provided smaller stagnation point heating than power-law shapes under the range of conditions investigated.

In order to study the shock-wave structure on power-law leading edges, a preliminary investigation was performed by Santos and Lewis. ${ }^{17}$ In their analysis, the shock-wave center or shock-wave "location" was defined from the inflection point in the shock-normal velocity profile. The shock-wave thickness was defined with the maximum-slope definition of shock thickness; for a given property $\phi$, which may be velocity, temperature density or pressure, the thickness was defined in a dimensional form in the shock-normal direction ${ }^{22}$ by

$$
\delta=\frac{\phi_{\max }-\phi_{\min }}{|d \phi / d \eta|_{\max }}
$$

where the maximum and minimum values of $\phi$ occur at $\eta= \pm \infty$. The term $|d \phi / d \eta|_{\max }$ represents the absolute value of the maximum gradient of $\phi$, regardless of the location within the shock wave where it occurs. $\eta$ represents the spatial coordinate. In this scenario, the velocity profile along the stagnation streamline was used not only to the shock-wave shape but also for the shock-wave thickness determination. It should be mentioned that, since each physical variable will have its own profile, each one would also have its own characteristic thickness. Therefore, the definition of thickness of a shock wave depends on the quantity $\phi$ that is being measured. $\mathrm{Grad}^{23}$ as well as Gilbarg and Paolucci ${ }^{24}$ emphasized that an objection to the definition given by Eq. (1) is that it depends on a purely local feature of the shock-wave profile, and that in the general case this measure of shock thickness is unrealistically small. In addition to that, Santos and Lewis ${ }^{17}$ observed that some velocity profiles presented no inflection point in the profiles along the stagnation streamline. A difference in the velocity profile behavior was observed for $n<2 / 3$ from that for $n \geqslant 2 / 3$. Consequently, due to the lack of inflection point on the velocity profile for some

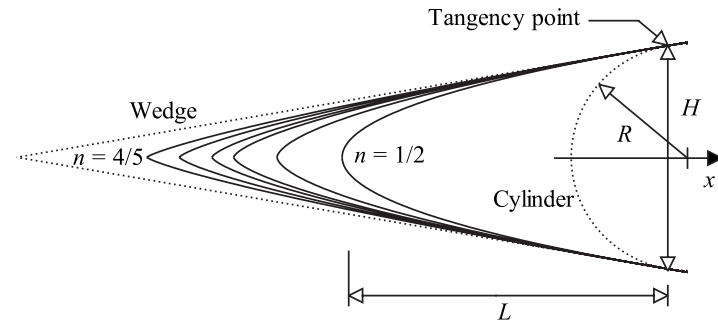

FIG. 1. Drawing illustrating the leading edge geometry.

cases, it was not possible to apply Eq. (1) for all cases investigated.

Based on recent interest in hypersonic waveriders for high-altitude/low-density applications, ${ }^{25-29}$ this paper extends the analysis presented by Santos and Lewis ${ }^{17}$ by computationally investigating the shock-wave structure over power-law leading edges by employing a procedure based on the physics of the particles. In this scenario, the primary goal is to assess the sensitivity of the shock-wave standoff distance, shock-wave thickness, and shock-wave shape to variations on the leading-edge shape and to compare them to the round shape (circular cylinder). Comparisons based on shock standoff distance are made to examine the benefits and disadvantages of using power-law shapes over round shapes.

The study at hand focuses on the low-density region in the upper atmosphere, where the nonequilibrium conditions are such as the traditional computational fluid dynamics (CFD) calculations are inappropriate to yield accurate results. In such a circumstance, the DSMC method will be employed to calculate the rarefied hypersonic twodimensional flow on the power-law shaped leading edges.

\section{LEADING-EDGE GEOMETRY DEFINITION}

In dimensional form, the body power-law shapes are given by the following expression:

$$
y=a x^{n},
$$

where $n$ is the power-law exponent and $a$ is the power-law constant which is a function of $n$.

The power-law shapes are modelled by assuming a sharp-edged wedge of half angle $\theta$ with a circular cylinder of radius $R$ inscribed tangent to this wedge. The power-law shapes, inscribed between the wedge and the cylinder, are also tangent to them at the same common point where they have the same slope angle. The circular cylinder diameter provides a reference for the amount of blunting desired on the leading edges. It was assumed a leading edge half angle of $10^{\circ}$, a circular cylinder diameter of $10^{-2} \mathrm{~m}$, and powerlaw exponents of $1 / 2,3 / 5,2 / 3,7 / 10,3 / 4$, and $4 / 5$. Figure 1 illustrates schematically this construction for the set of power-law leading edges investigated.

From geometric considerations, the common body height $H$ at the tangency point is equal to $2 R \cos \theta$. The power-law constant $a$, obtained by the matching slope on the wedge, circular cylinder and power-law body at the tangency 
point, and the body length $L$, from the nose to the tangency point in the axis of symmetry, are given by the following expressions:

$$
\begin{aligned}
a & =\frac{\left(\frac{R^{2}}{1+\tan ^{2} \theta}\right)^{(1-n) / 2}}{\left(\frac{n}{\tan \theta}\right)^{n}}, \\
L & =\frac{n R \cos ^{2} \theta}{\sin \theta}=\frac{n R}{\tan \theta \sqrt{1+\tan ^{2} \theta}} .
\end{aligned}
$$

It was assumed that the power-law leading edges are infinitely long but only the length $L$ is considered in the simulation since the wake region behind the power-law bodies is not of interest in this investigation.

\section{COMPUTATIONAL TOOL}

It has been firmly established that the most successful numerical technique for modelling complex flows in the transitional flow regime is the direct simulation Monte Carlo (DSMC) method developed by Bird. ${ }^{30}$ The DSMC method models the flow as being a collection of discrete particles, each one with a position, velocity, and internal energy. The state of the particles is stored and modified with time as the particles move, collide, and undergo boundary interactions in simulated physical space. The molecular motion and the intermolecular collisions are uncoupled over the small time step used to advance the simulation. Therefore, the time step should be sufficiently small in comparison to the local mean collision time. $^{31,32}$

The molecular collisions are modelled by the variable hard sphere (VHS) molecular model, ${ }^{33}$ and by the no time counter (NTC) collision sampling technique. ${ }^{34}$ The energy exchange between kinetic and internal modes is controlled by the Larsen-Borgnakke statistical model. ${ }^{35}$ The conditions used for the present investigation are for low-energy flow where there are no chemical reactions. Therefore, simulations are performed using a nonreacting gas model for air consisting of two chemical species, $\mathrm{N}_{2}$ and $\mathrm{O}_{2}$. Energy exchanges between translational, rotational, and vibrational modes are considered. The rate of rotational and vibrational relaxation are dictated by collision numbers $Z_{R}$ and $Z_{V}$, respectively. Constant collision numbers of 5 and 50 are given for rotation and vibration, respectively.

\section{COMPUTATIONAL FLOW DOMAIN AND GRID}

In order to easily account for particle-particle collisions, the flowfield is divided into an arbitrary number of regions, which are subdivided into computational cells. The cells are further subdivided into four subcells, two subcells/cell in each coordinate direction. In this fashion, the cell provides a convenient reference sampling of the macroscopic gas properties, while the collision partners are selected from the same subcell for the establishment of the collision rate. The linear dimensions of the cells should be of the order of or even smaller than the local mean free path. ${ }^{36,37}$ Close to the body surface, cell spacing normal to the body should be also of the



FIG. 2. Schematic view of the computational domain.

order of a third of the local mean free path. If the cell size near the body surface is too large, then energetic molecules at the far edge of the cell are able to transmit momentum and energy to molecules immediately adjacent to the body surface. This leads to overprediction of both the surface heat flux and the aerodynamic forces on the body that would occur in the real gas. ${ }^{38}$

The computational domain used for the calculation is made large enough so that body disturbances do not reach the upstream and side boundaries, where freestream conditions are specified. The boundary conditions are specified in terms of the behavior of the individual molecules rather than the distribution function. Four boundary conditions are specified. A schematic view of the computational domain is depicted in Fig. 2. According to Fig. 2, side 1 is defined by the body surface. Diffuse reflection with complete thermal accommodation is the condition applied to this side. An advantage of the flow symmetry is taken into account, and molecular simulation is applied to one-half of a full configuration. Thus, side 2 is a plane of symmetry. In such a boundary, all flow gradients normal to the plane are zero. At the molecular level, this plane is equivalent to a specular reflecting boundary. Side 3 is the freestream side through which simulated molecules enter and exit. Finally, the flow at the downstream outflow boundary, side 4 , is a boundary with a vacuum or with a set of molecules crossing into the computational domain. The vacuum option is suitable for an outflowing gas with a highly supersonic velocity component across such a boundary, since there are virtually no upstream moving molecules in a flow with Mach number greater than about $3 .{ }^{39}$ Hence, vacuum is the prescribed boundary condition for this side. Usually, the upstream extent of the interference due to this unrealistic boundary condition is determined by increasing the body length.

Numerical accuracy in the DSMC method depends on the grid resolution chosen as well as on the number of particles per computational cell. Both effects were investigated to determine the number of cells and the number of particles required to achieve grid independence solutions. A grid independence study was made with three different structured meshes in each coordinate direction, $\xi$ - and $\eta$-direction as shown in Fig. 2. The effect of altering the cell size in the $\xi$-direction was investigated with grids of 35 (coarse), 70 
(standard), and 105 (fine) cells, and 50 cells in the $\eta$-direction for power-law exponent of $1 / 2$. In an analogous fashion, an examination was made in the $\eta$-direction with grids of 25 (coarse), 50 (standard), and 75 (fine) cells, and 70 cells in the $\xi$-direction for power-law exponent of $1 / 2$. Each grid was made up of nonuniform cell spacing in both directions. The effect (not shown) of changing the cell size in both directions on the heat transfer, pressure and skin friction coefficients was rather insensitive to the range of cell spacing considered, indicating that the standard grid, $70 \times 50$ cells, for the power-law shape defined by $n=1 / 2$ is essentially grid independent. A similar procedure was performed for the other cases investigated. For instance, results indicated that a grid of $80 \times 50$ and $90 \times 50$ for power-law exponents of $2 / 3$ and $4 / 5$, respectively, were considered fully independent. Of particular interest is the number of cells in the $\eta$-direction for the power-law cases investigated. It should be emphasized that, even though the number of cells is the same in the $\eta$-direction, the computational domain size is different for each one of the cases. In this way, side 2 shown in Fig. 2 corresponds, for instance, to 8,6 , and $5 \lambda_{\infty}$ for power-law exponents of $1 / 2,2 / 3$, and $4 / 5$, respectively, where $\lambda_{\infty}$ is the freestream mean free path.

In a second stage of the grid independence investigation, a similar examination was made for the number of molecules. The standard grid for power-law exponent of $1 / 2$, $70 \times 50$ cells, corresponds to, on average, a total of 121000 molecules. Two new cases using the same grid were investigated. These two new cases correspond to 108000 and 161000 molecules in the entire computational domain. As the three cases presented approximately the same results for the heat transfer, pressure and skin friction coefficients, hence the standard grid with a total of 121000 molecules was considered enough for the computation of the aerodynamic surface quantities. Again, a similar procedure was performed for the other cases investigated. As a result, a total of 126000 and 144000 simulated molecules were used for power-law exponent cases of $2 / 3$ and 4/5, respectively.

In order to obtain a more uniform distribution of simulated particles per cell, a variable time-step method was employed. Advantages of implementing the variable time-step scheme are to reduce both the simulated particle numbers and the number of interactions for transient period towards steady state, when sampling normally begins in DSMC. In this fashion, time step changed from $2.8 \times 10^{-11}$ to 1.4 $\times 10^{-6} \mathrm{~s}$. For the cases investigated, 20000 time steps were used in order to reach the steady state conditions and 100000 independent samples, with 4 time steps between independent samples.

\section{FREESTREAM AND FLOW CONDITIONS}

The freestream flow conditions used for the numerical simulation of flow past the leading edges are those given by Santos and Lewis ${ }^{12}$ and summarized in Table I and the gas properties $^{30}$ are shown in Table II.

The freestream velocity $V_{\infty}$ is assumed to be constant at $3.56 \mathrm{~km} / \mathrm{s}$, which corresponds to freestream Mach number $M_{\infty}$ of 12 . The wall temperature $T_{w}$ is assumed constant at
TABLE I. Freestream and flow conditions.

\begin{tabular}{lll}
\hline \hline \multicolumn{1}{c}{ Parameter } & Value & Unit \\
\hline Temperature $\left(T_{\infty}\right)$ & 220.0 & $\mathrm{~K}$ \\
Pressure $\left(p_{\infty}\right)$ & 5.582 & $\mathrm{~N} / \mathrm{m}^{2}$ \\
Density $\left(\rho_{\infty}\right)$ & $8.753 \times 10^{-5}$ & $\mathrm{~kg} / \mathrm{m}^{3}$ \\
Viscosity $\left(\mu_{\infty}\right)$ & $1.455 \times 10^{-5}$ & $\mathrm{~N} \mathrm{~s} / \mathrm{m}^{2}$ \\
Number density $\left(n_{\infty}\right)$ & $1.8209 \times 10^{21}$ & $\mathrm{~m}^{-3}$ \\
Mean free path $\left(\lambda_{\infty}\right)$ & $9.03 \times 10^{-4}$ & $\mathrm{~m}$ \\
\hline \hline
\end{tabular}

$880 \mathrm{~K}$. This temperature is chosen to be representative of the surface temperature near the stagnation point and is assumed to be uniform over the bodies. It is important to mention that the surface temperature is low compared to the stagnation temperature of the air. This assumption seems to be reasonable since practical surface material will probably be destroyed if surface temperature is allowed to approach stagnation temperature.

The freestream Knudsen number, $\mathrm{Kn}_{\infty}$, corresponds to 0.0903, where the characteristic dimension was defined as being the diameter of the reference circular cylinder (see Fig. 1). Finally, the freestream Reynolds number $\operatorname{Re}_{\infty}$ by unit meter is 21455 .

\section{COMPUTATIONAL PROCEDURE}

The problem of predicting thickness, shape, and location of detached shock waves has been stimulated by the necessity for blunt noses and leading edges configurations designed for hypersonic flight in order to cope with the aerodynamic heating. In addition, the ability to predict thickness, shape and location of shock waves is of primary importance in analysis of aerodynamic interference. Furthermore, the knowledge of the shock-wave displacement is also especially important in waveriders, ${ }^{1}$ since these hypersonic configurations usually rely on shock-wave attachment at the leading edge to achieve their high lift-to-drag ratio at high-lift coefficient.

In the present account, the shock-wave structure, defined by thickness, detachment, and shape of the shock wave, is predicted by employing a procedure based on the physics of the particles. In this respect, the flow is assumed to consist of three distinct classes of molecules: class I molecules denote those molecules from the freestream that have not been affected by the presence of the leading edge; class II molecules are those molecules that have collided and been reflected from the body surface at some time in their past history; and finally, those molecules that have been indirectly affected by

TABLE II. Gas properties.

\begin{tabular}{llll}
\hline \hline \multicolumn{1}{c}{ Parameter } & $\mathrm{O}_{2}$ & $\mathrm{~N}_{2}$ & Unit \\
\hline Molecular mass & $5.312 \times 10^{-26}$ & $4.65 \times 10^{-26}$ & $\mathrm{~kg}$ \\
Molecular diameter & $4.010 \times 10^{-10}$ & $4.11 \times 10^{-10}$ & $\mathrm{~m}$ \\
Mole fraction & 0.237 & 0.763 & \\
Viscosity index & 0.77 & 0.74 & \\
\hline
\end{tabular}




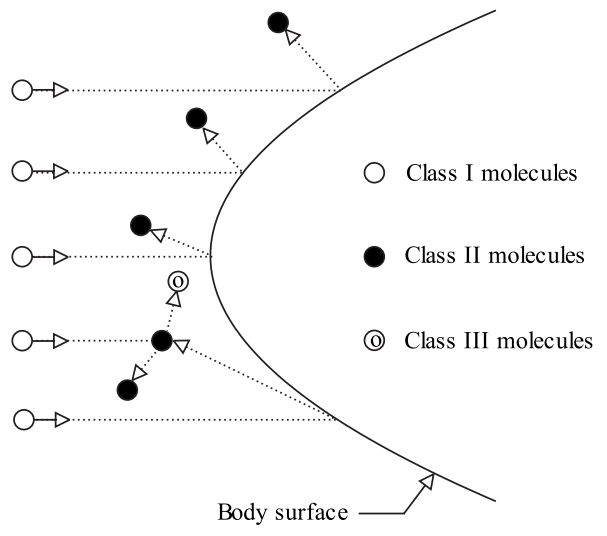

FIG. 3. Drawing illustrating the classification of molecules.

the presence of the body are defined as class III molecules. Figure 3 illustrates the definition for the molecular classes.

It is assumed that the class I molecule changes to class III molecule when it collides with either class II or class III molecules. Class I or class III molecules are progressively transformed into class II molecule when it interacts with the body surface. Also, a class II molecule remains class II independently of subsequent collisions and interactions. Hence, the transition from class I molecules to class III molecules may represent the shock wave, and the transition from class III to class II may define the boundary layer.

A typical distribution of class III molecules along the stagnation streamline for blunt leading edges is displayed in Fig. 4 along with the definition used to determine the thickness, displacement and shape of the shock wave. In this figure, $X$ is the distance $x$ along the stagnation streamline, normalized by the freestream mean free path $\lambda_{\infty}$, and $f_{\text {III }}$ is the ratio of the number of molecules for class III inside the cell to the total number of molecules inside the same cell. Also, the flow direction is from left to right side, as defined in Fig. 2.

In a rarefied flow, the shock wave has a finite region that depends on the transport properties of the gas, and can no longer be considered as a discontinuity obeying the classical

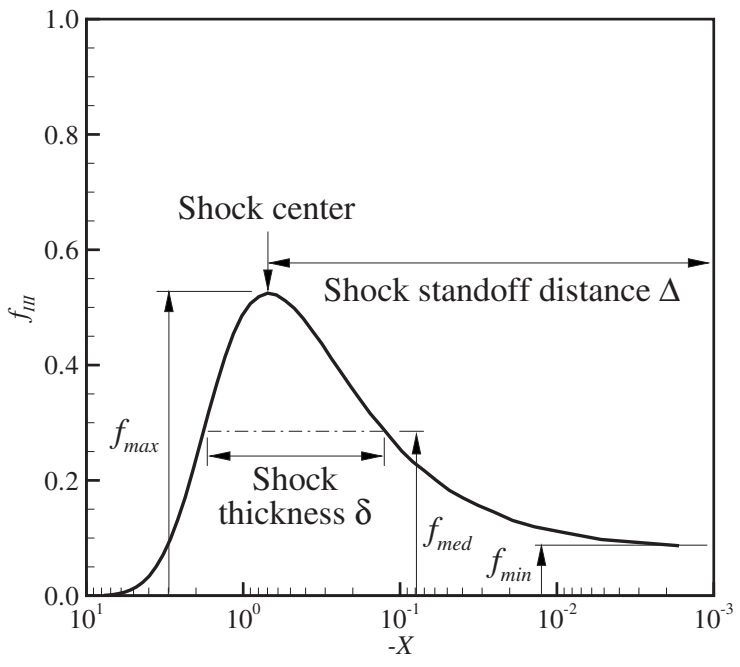

FIG. 4. Drawing illustrating the shock wave structure.
Rankine-Hugoniot relations. In this sense, the shock standoff distance $\Delta$ is defined as being the distance between the shock wave center and the nose of the leading edge along the stagnation streamline. As shown in Fig. 4, the center of the shock wave is defined by the station that corresponds to the maximum value for $f_{\text {III }}$. The shock-wave thickness $\delta$ is defined by the distance between the stations that correspond to the mean value for $f_{\text {III }}$. Finally, the shock wave "location" is determined by the coordinate points given by the maximum value in the $f_{\mathrm{III}}$ distribution along the lines departing from the body surface, i.e., $\eta$-direction as shown in Fig. 2.

The molecule classification that has been adopted here was first presented by Lubonski ${ }^{40}$ in order to study the hypervelocity Couette flow near the free molecular flow regime. Lubonski ${ }^{41}$ divided the gas into three classes of molecules: "freestream," "reflected from the boundary" and "scattered." Later, for the purpose of flow visualization, Bird $^{41}$ applied a similar scheme of classification by identifying the classes by colors: blue for class I, red for class II, and yellow for class III molecules.

\section{COMPUTATIONAL RESULTS AND DISCUSSION}

This section focuses on the effects that take place on the shock-wave structure due to variations on the leading-edge shape. In this scenario, the purpose of this section is to discuss and to compare differences in the thickness, displacement, and shape of the shock wave due to variations on the power-law exponent $n$. Nevertheless, having computed molecular class properties over a wide range of simulation parameters, it proves instructive to summarize first the major features of the results related to the class of molecules.

\section{A. Distribution of molecular classes}

The distribution of molecules for the three classes along the stagnation streamline is demonstrated in Figs. 5(a) and 5(b) for power-law exponent $n$ of $1 / 2$ and 4/5, respectively. In this set of figures, $f_{\mathrm{I}}, f_{\mathrm{II}}$, and $f_{\mathrm{III}}$ are the ratio of the number of molecules for class I, II, and III, respectively, to the total amount of molecules inside each cell along the stagnation streamline, and $X$ is the distance $x$ along the stagnation streamline normalized by the freestream mean free path $\lambda_{\infty}$. Again, the flow direction is from left to right as defined in Fig. 2. The distributions for the other power-law cases, intermediate to those shown in Figs. 5(a) and 5(b), will not be shown.

Of great significance in these figures is the behavior of the molecules around sharp and blunt leading edges. It should be noticed that molecules from freestream, represented by class I molecules, do not reach the nose of the leading edge for the $n=1 / 2$ case. This behavior is shown in Fig. 5(a), which represents a blunt leading edge. In contrast, molecules from freestream collide with the nose of the leading edge for the $n=4 / 5$ case, even after the establishment of the steady state. This situation is illustrated in Fig. 5(b), which represents a sharp leading edge.

Before proceeding with the shock wave structure analysis, it is desirable to present the distribution of the molecular classes adjacent to the leading edge surfaces. In this way, 




(a)

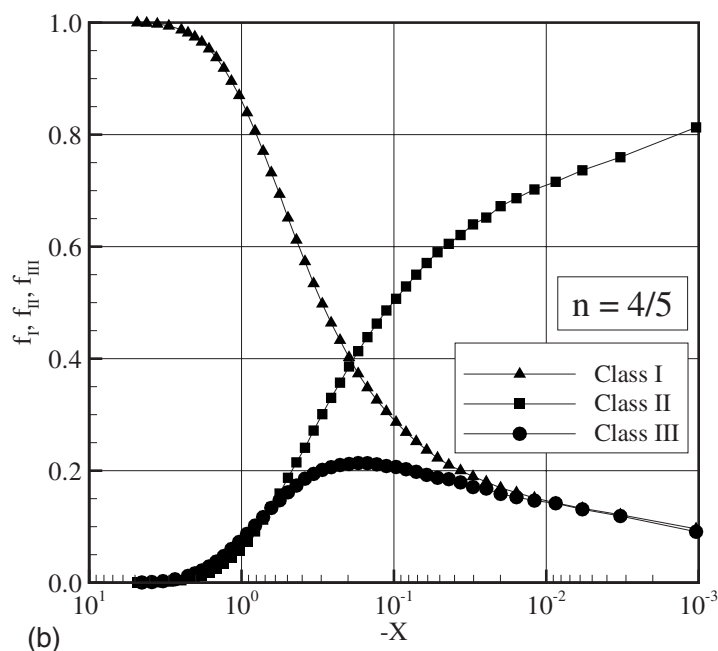

FIG. 5. Distributions of molecules for classes I, II, and III along the stagnation streamline for power law exponents of (a) $1 / 2$ and (b) 4/5.

particular attention is paid to the power-law exponents of $1 / 2$ and $4 / 5$, the bluntest and the sharpest leading edges investigated.

Molecular class contours are displayed in Figs. 6(a)-6(c) for class I, II, and III, respectively. In this set of diagrams, molecular class distribution for power-law exponent of $1 / 2$, displayed on the upper part of the figures, is compared to that for power-law exponent of $4 / 5$, shown on the lower part of the figures.

Important features can be observed in Figs. 6(a)-6(c). From Fig. 6(a), it is seen that the molecules for class I reaches the surface of the leading edge only at the stagnation region for power-law exponent of $4 / 5$, in contrast to powerlaw exponent of $1 / 2$. As a result, the heat flux to the nose of the leading edge for the $n=4 / 5$ case is more severe as that for the $n=1 / 2$ case, as shown by Santos and Lewis. ${ }^{12}$

The region defined by class II molecules is related to the boundary layer on the body surface. In this respect, it is clearly seen from Fig. 6(b) that the boundary layer for the $n=1 / 2$ case is larger than that for the $n=4 / 5$ case, as would be expected since the leading edge defined by the $n=4 / 5$ case is more streamlined than that for the $n=1 / 2$ case.

Class III molecules represent the effect of the presence



(a)

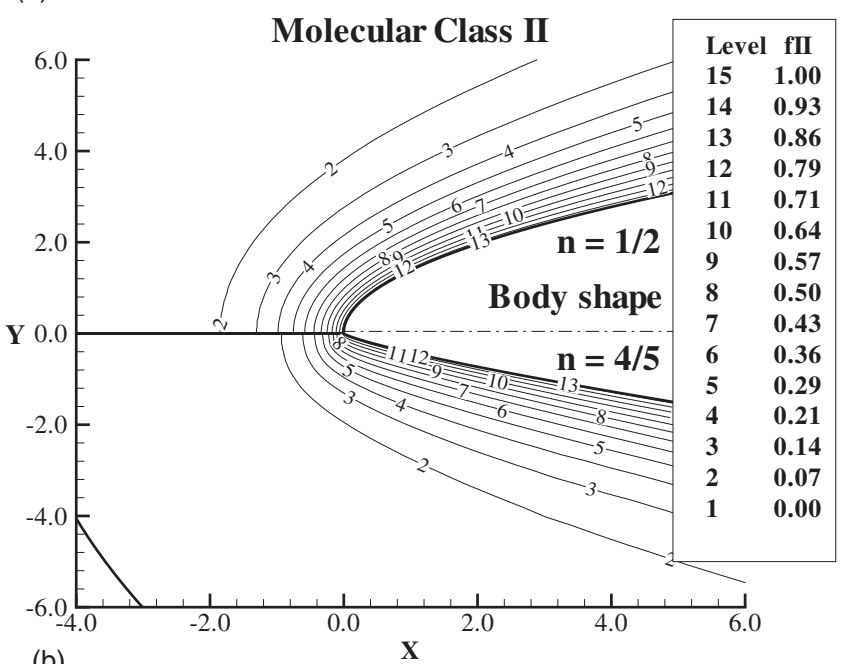

(b)

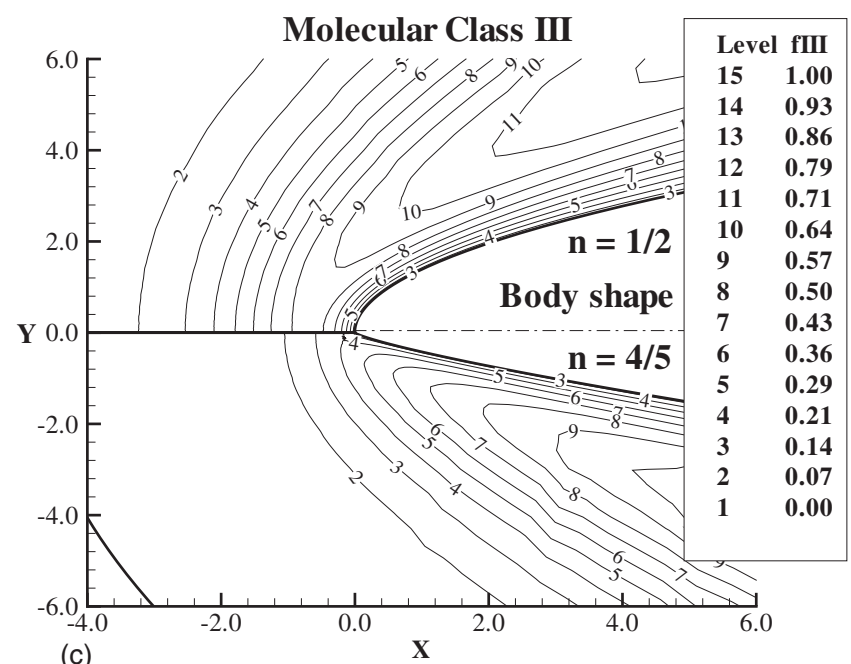

(c)

FIG. 6. Comparison of molecular class distribution adjacent to the body surface for power law exponents of $1 / 2$ (upper part) and 4/5 (lower part). Class (a) I, (b) II, and (c) III.

of the leading edge that is propagated in the off-body direction. According to Fig. 6(c) the upstream disturbance along the stagnation streamline is more significant for blunt leading edge than that for sharp leading edge, as would be expected too. 
TABLE III. Dimensionless shock-wave standoff distance $\Delta / \lambda_{\infty}$ for the power-law leading edges.

\begin{tabular}{ccccccc}
\hline \hline$n$ & $1 / 2$ & $3 / 5$ & $2 / 3$ & $7 / 10$ & $3 / 4$ & $4 / 5$ \\
\hline$\Delta / \lambda_{\infty}$ & 0.678 & 0.459 & 0.343 & 0.305 & 0.231 & 0.165 \\
\hline \hline
\end{tabular}

\section{B. Shock-wave standoff distance}

Referring to the definition introduced in Fig. 4, the shock standoff distance $\Delta$ can be observed in Fig. 5 for the powerlaw leading edges shown. The calculated shock standoff distance $\Delta$, normalized by the freestream mean free path $\lambda_{\infty}$ is tabulated in Table III for the cases investigated. It is apparent from these results that there is a discrete shock standoff distance for the cases shown. As would be expected, the shock standoff distance decreases with increasing the power-law exponent $n$. As a reference, the shock-wave standoff distance for the bluntest leading edge case, $n=1 / 2$, is around 4.1 times larger than that for $n=4 / 5$.

According to Santos and Lewis, ${ }^{18}$ the round leading edge (circular cylinder), shown in Fig. 1, provides a shock detachment $\Delta / \lambda_{\infty}$ of 1.645 at the same flow conditions. For comparison purposes, this value is about 2.4 and 10 times larger than the cases corresponding to $n$ of $1 / 2$ and $4 / 5$, respectively. The results confirm the expectation that the shock standoff distance for sharp leading edge is smaller than that for blunt leading edge. In fact, power-law leading edges behave as if they had a sharper profile than the representative circular cylinder.

It should be emphasized that shock standoff distance becomes important in hypersonic vehicles such as waveriders, which depend on the leading edge shock attachment to achieve their high lift-to-drag ratio at high lift coefficient. Nonetheless, smaller shock detachment distance is associated with a higher heat load to the nose of the body. The heat transfer coefficient $C_{h}\left(=2 q_{w} / \rho_{\infty} V_{\infty}^{3}\right)$ at the stagnation point for power-law leading edges with $n$ of $1 / 2$ and $4 / 5$ is 1.5 and 3.4 times larger than the heat transfer coefficient for the circular cylinder $^{42}$ at the same conditions.

\section{Shock-wave thickness}

Based on the definition of the shock-wave thickness shown in Fig. 4, the shock-wave thickness $\delta$ along the stagnation streamline can be obtained from Fig. 5 for the leading edge shapes. As a result of the calculation, Table IV tabulates the shock-wave thickness $\delta$, normalized by the freestream mean free path $\lambda_{\infty}$, for the cases investigated.

Referring to Table IV, the shock-wave thickness for the bluntest leading edge case, $n=1 / 2$, is around 3 times larger than that for $n=4 / 5$.

TABLE IV. Dimensionless shock-wave thickness $\delta / \lambda_{\infty}$ for the power-law leading edges.

\begin{tabular}{ccccccc}
\hline \hline$n$ & $1 / 2$ & $3 / 5$ & $2 / 3$ & $7 / 10$ & $3 / 4$ & $4 / 5$ \\
\hline$\delta / \lambda_{\infty}$ & 1.586 & 1.110 & 0.887 & 0.790 & 0.642 & 0.524 \\
\hline \hline
\end{tabular}

In what follows, the reference circular cylinder ${ }^{42}$ provides a much larger shock thickness, $\delta / \lambda_{\infty}$ of 3.350 at the same flow conditions. Compared to power-law leading edges, this value is about 2.1 and 6.4 times larger than the cases corresponding to power-law exponents of $1 / 2$ and $4 / 5$, respectively.

\section{Shock-wave shape}

The shock-wave shape, defined by the shock-wave center, is obtained by calculating the position that corresponds to the maximum $f_{\text {III }}$ in the $\eta$-direction along the body surface (see Fig. 4).

Figures $7(a)-7(c)$ illustrate the shock-wave shape at the vicinity of the leading edge noses for power-law shapes defined by $n$ of $1 / 2,2 / 3$, and $3 / 4$, respectively. In this set of plots, $X$ and $Y$ are the Cartesian coordinates $x$ and $y$ normalized by $\lambda_{\infty}$.

It was pointed by Lees and Kubota ${ }^{4}$ that flow similarity is possible for a class of bodies of the form $x^{n}$. In the more general case for $0<n<1$, the shock wave grows like $x^{m}$. When $n$ grows from zero, $m$ begins by keeping the constant value $m=2 / 3$, and if $n$ keeps on growing towards one, $m$ remains equal to $n$. The similarity solutions are obtained by assuming the hypersonic slender body approximations. ${ }^{21}$ Consequently, they are not valid near the nose of the leading edge where the approximations are violated. At or near the nose, the surface slope, the curvature and higher derivatives are infinite and, therefore, the similarity solutions breakdown.

It is very encouraging to observe that the Lees and Kubota $^{4}$ findings were obtained by considering the continuum assumptions. Although the present account considers shock wave in the transitional flow regime, i.e., between the continuum flow regime and the free molecular flow regime, it becomes instructive to verify if the shock-wave shape on power-law body in this flow regime follows the same behavior of that in the continuum flow regime. Hence, a fitting process is performed over the coordinate points yielded by DSMC simulations in order to approximate the shock-wave shapes by the following form:

$$
y=A(x+B)^{m},
$$

where $A$ is the power-law constant of the curve fit, $B$ is the distance from the nose of the leading edge, and $m$ is the power-law exponent of the curve fit, i.e., the shock-wave power-law exponent.

Curve-fit solutions for shock-wave shape over powerlaw leading edges given by $n$ of $1 / 2$ and $2 / 3$ are displayed in Figs. 8(a) and 8(b). In this set of diagrams, $n$ represents the shock wave yielded by the DSMC solution and $m$ the curvefit solution. In addition to that, the curve-fit solutions shown were obtained according to Eq. (5) by three different forms: (1) $A$ and $B$ were found by keeping $m$ equal to the body shape, $m=n$; (2) $A, B$ and $m$ were found in order to obtain the best fit; finally (3) $A$ and $B$ were found by keeping $m$ $=2 / 3$, the exponent that it is expected that the shock wave would grow, according to the theory (Lees and Kubota ${ }^{4}$ ). Moreover, the fitting process is performed over the coordi- 



FIG. 7. Shock-wave shape on body power-law exponent of (a) $1 / 2$, (b) $2 / 3$, and (c) $3 / 4$.

nate points located far from the stagnation region (say $X>3.0$ ), where it is expected that the blunt nose effects are no more important.

Referring to Figs. 8(a) and 8(b), the curves defined by $m=0.670$ and 0.764 represent the second form of the curve
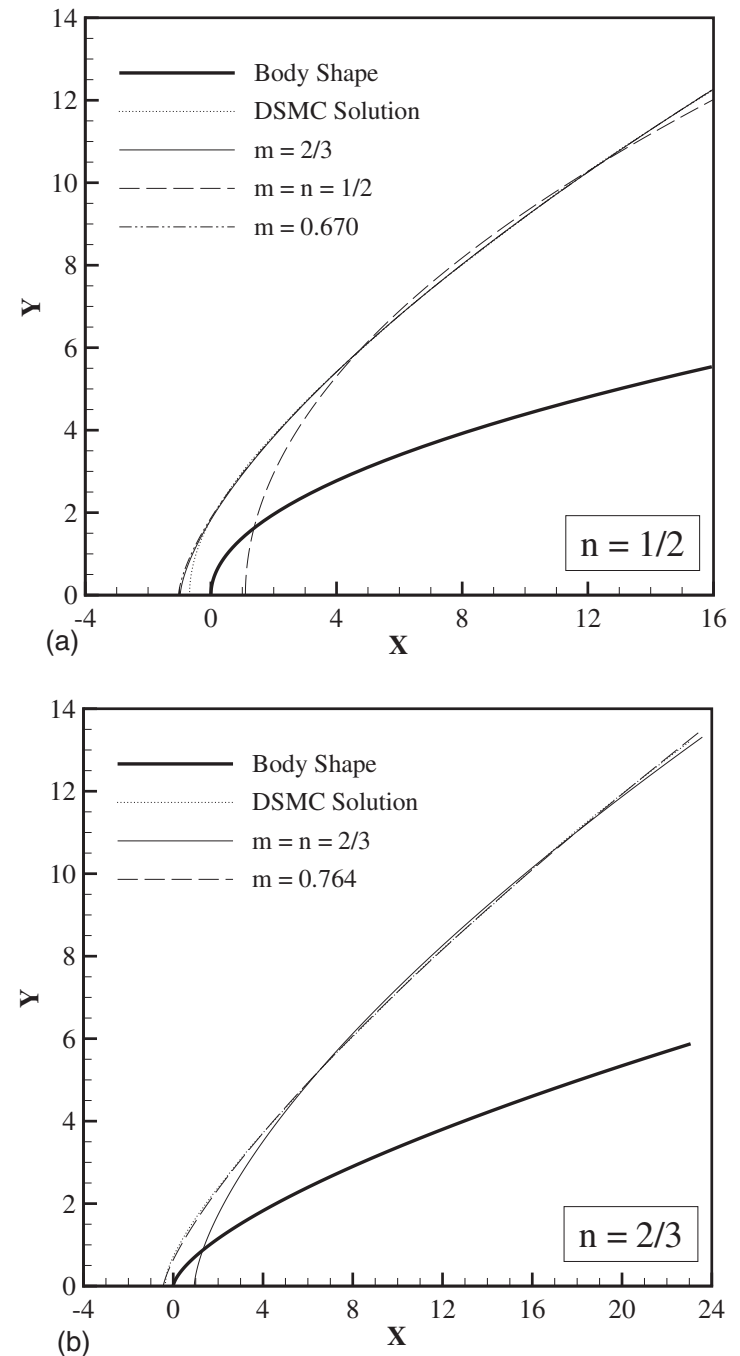

FIG. 8. Shock-wave shape curve fit on body power-law exponent of (a) $1 / 2$, and (b) $2 / 3$.

fit solutions mentioned above. It is apparent from this set of figures that the curve-fit solutions present a good agreement, by visual inspection, with those solutions provided by the DSMC simulation. Nevertheless, as the maximum absolute error between the DSMC solutions and the curve fit solutions are calculated for coordinate points located at $X>3.0$, it is found that the curve fit obtained by the second form of the fitting process presents a slightly better fit, i.e., when $A, B$, and $m$ were found in order to yield the best solution. The error is less then $1.0 \%$ for the curves shown in Figs. 8(a) and 8(b).

According to Fig. 8(a), it is clearly seen that the curve-fit solution given by $m=n=1 / 2$ do not match the shock-wave shape obtained by the DSMC simulation, as predicted by the hypersonic small-disturbance theory. In contrast, the two other curve-fit solutions, $m=2 / 3$ and $m=0.670$ present an excellent agreement with those solutions provided by the DSMC simulation. Once again, the curve-fitted solution deviates from the DSMC solution close to the nose of the leading edge, as would be expected.

Curve-fit solutions for shock-wave shape over powerlaw leading edges given by $n$ of $3 / 4$ and $4 / 5$ are displayed in 

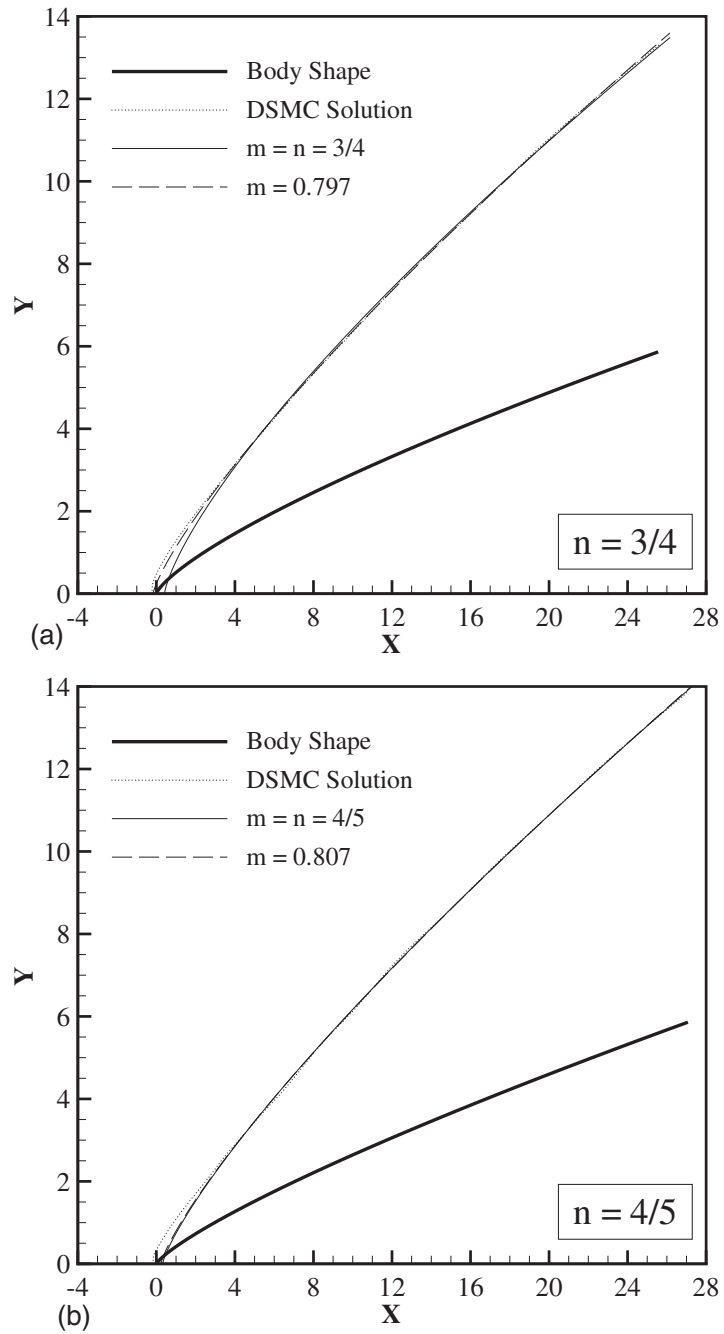

FIG. 9. Shock-wave shape curve fit on body power-law exponent of (a) 3/4 and (b) $4 / 5$.

Figs. 9(a) and 9(b). For comparison purpose, two forms of the curve fit were considered in defining the shock shape: (1) $A, B$, and $m$ were found to provide the best curve-fit solutions, and (2) $A$ and $B$ were found by keeping $m=n$ for $n>2 / 3$ cases, where again $n$ and $m$ account for body and shock-wave power-law exponents, respectively. It is apparent from this set of diagrams that the curve-fit solutions present a good agreement, by visual inspection, with those solutions provided by the DSMC simulation.

In general, as indeed is clear from the DSMC results, the solutions are in good qualitative agreement with the Lees and Kubota $^{4}$ findings in the sense that the shock-wave shape follows the shape of the body for body power-law exponent $n>2 / 3$. It should be emphasized that the curve-fit exponents are very sensitive to the number of coordinate points, which define the shock wave shape, used in the fitting process.

At this point it is worth taking a closer look at the shockwave shape at the vicinity of the leading-edge nose. In this region, the shock-wave shape is not correctly predicted by the theoretical solutions, since the hypersonic slender body approximations are violated close to or at the nose of the leading edges, as explained earlier.

Before proceeding with the analysis, it is desirable to point out some interesting features, presented in the literature, related to the shock-wave shape at the vicinity of the leading-edge nose for blunt body in the continuum flow regime.

It is usually accepted without question that curved shock wave supported by a blunt-nosed body introduces vorticity in the flowfield between the body and the shock wave. In this context, by investigating two-dimensional, inviscid, and perfect gas assumptions power-law leading edges, therefore in the continuum regime, O'Brien ${ }^{43}$ observed that vorticity immediately behind a power-law shock wave approaches minus infinity for power-law exponents $m>2 / 3$. Consequently, if power-law body $\left(\propto x^{n}\right)$ produces power-law shock wave $\left(\propto x^{m}\right)$ at the vicinity of the leading-edge nose, then for the body exponent range of $n>2 / 3$, the shock-wave exponent should be $m<2 / 3$ in order to avoid infinity vorticity behind the shock wave.

According to Mason and Lee, ${ }^{3}$ the radius of curvature for power-law shapes, obtained from the general formula for the longitudinal radius of curvature, goes to infinity at the nose $(x \rightarrow 0)$ for values of $0<n<1 / 2$, approaches zero for values of $1 / 2<n<1$, and is finite for $n=1 / 2$. For the $n=1 / 2$ case, the radius of curvature is equal to $a^{2} / 2$, where $a$ is the body power-law constant defined in Eq. (2).

From Schlieren photographs obtained for flat plates in hypersonic flow, Vas et al. ${ }^{44}$ found that a power-law shock wave with exponent of 0.49 offered the best fit on a flat plate with a square leading edge, and a power-law shock wave with exponent of 0.50 provided the best fit for a flat plate with a round leading edge. McCarthy et al. ${ }^{45}$ reported an experimental study of wakes behind a circular cylinder in supersonic flow and also found a power-law shock-wave with exponent of 0.516 . Zapata et al. $^{46}$ studied high Mach number and low Reynolds number flow over a twodimensional circular cylinder experimentally, and identified best fits power-law shock-waves with exponents of 0.517 and 0.494 for an uncooled and cooled model, respectively. These experiments represent flows at conditions and geometries that are very different from those chosen here, but they indicated a tendency towards parabolic shock waves. This supports the conclusion that the exponent of $1 / 2$ is preferred for the shock-wave shape near the stagnation streamline for blunt bodies in the continuum flow regime.

Selected in order to elucidate the requirements posed on the shock-wave shape at the vicinity of the leading-edge nose, these discussed features suggest that, at least at first sight, the shock-wave shape for power-law blunt body in the present account might be a power-law shape that grows with $m=0.5$ close to the stagnation streamline. In this sense, vorticity might be satisfied and the radius of curvature would be finite.

With this perspective in mind, a fitting process is performed over the coordinate points yielded by DSMC simulations in order to approximate the shock-wave shapes, at the vicinity of the leading-edge nose, according to Eq. (5) with $m=0.5$. Figures $10(\mathrm{a})-10$ (c) illustrate the curve-fit solutions for $n$ of $1 / 2,2 / 3$, and $3 / 4$, respectively.

Referring to Figs. 10(a)-10(c), it is clearly noticed that the curve-fit solutions present an excellent agreement with 


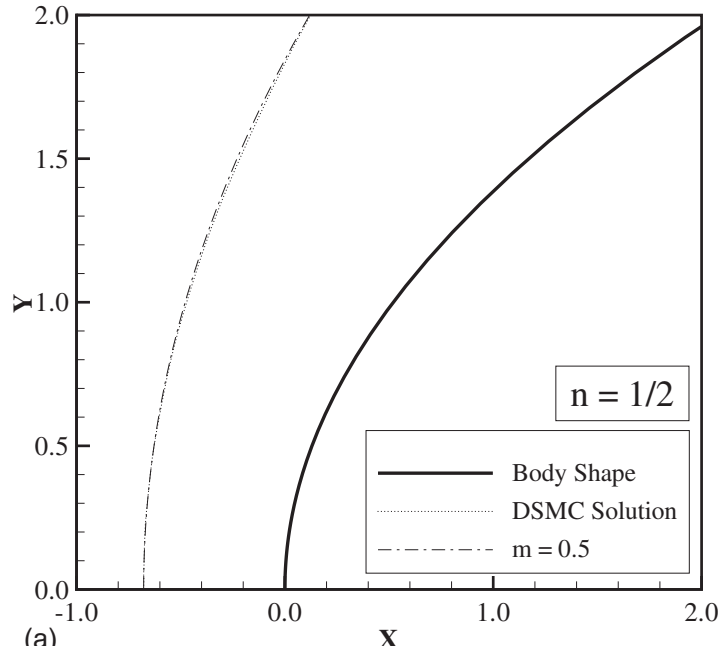

(a)

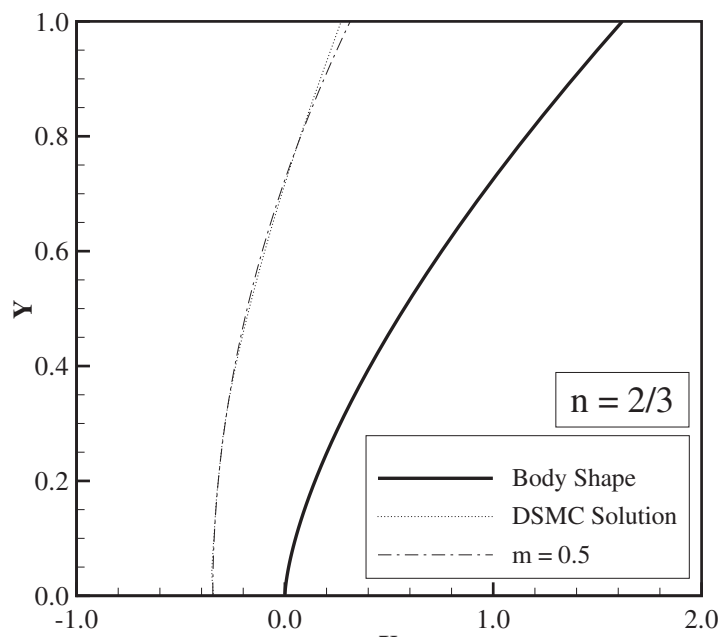

(b)

$\mathbf{X}$

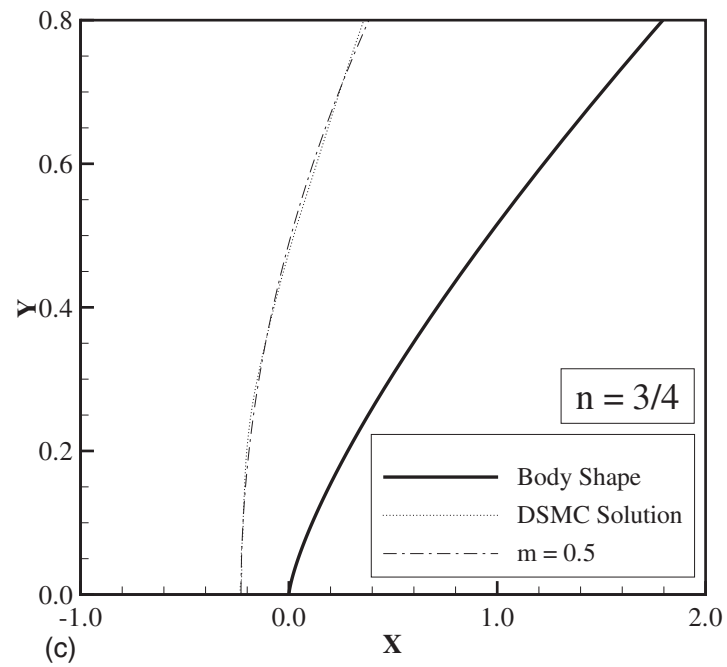

FIG. 10. Shock-wave shape curve fit, at the vicinity of the leading-edge nose, on body power-law exponent of (a) $1 / 2$, (b) $2 / 3$, and (c) $3 / 4$.

those solutions provided by the DSMC simulation. As a result, the shock-wave shape at the vicinity of the leading-edge nose is described by a power-law form with exponent $m=0.5$. Therefore, the qualitative findings regarding shockwave shape in the continuum flow regime seem to hold for the simulation results investigated here in the transitional flow regime.
It should also be mentioned in this context that no unique power-law exponent has been found that describes the shock-wave shape in the total length of the leading edges investigated. The results indicate that the shock-wave shape is best represented by at least two power-law exponents $m$. One of them is applied to the region near the nose of the leading edge, where the effects of the blunt nose are important. The other one represents the region where the bluntness effects are no longer important, as predicted by the classical analytical solution.

\section{CONCLUDING REMARKS}

This study applies the direct simulation Monte Carlo (DSMC) method to assess the impact on the shock-wave structure due to variations on the shape of power-law leading edges. The calculations provided information concerning the nature of the shock-wave shape, shock-wave thickness, and shock-wave detachment distance resulting from variations on the body shape for the idealized situation of two-dimensional hypersonic rarefied flow.

The computational results indicated that the shock-wave shape grows like $x^{m}$ for power-law leading edges. It was found that, at the vicinity of the nose, the shock-wave powerlaw exponent is $1 / 2$. In addition, the shock-wave shape confirmed the finding predicted by the hypersonic small disturbance theory in that the shape of the shock wave follows the shape of the body far from the nose of the leading edge, provided the body power-law exponent is larger than $2 / 3$.

\section{ACKNOWLEDGMENTS}

The author wishes to acknowledge the support yielded by Dr. F. Fachini from the Combustion and Propulsion Laboratory at the National Institute for Space Research (INPE), offering valuable discussions and suggestions during the analysis of the DSMC results.

${ }^{1}$ T. R. F. Nonweiler, "Aerodynamic problems of manned space vehicles," J. R. Aeronaut. Soc. 63, 521 (1959).

${ }^{2}$ M. J. Gillum and M. J. Lewis, "Experimental results on a Mach 14 waverider with blunt leading edges," J. Aircr. 34, 296 (1997).

${ }^{3}$ W. H. Mason and J. Lee, "Aerodynamically blunt and sharp bodies," J. Spacecr. Rockets 31, 378 (1994).

${ }^{4}$ L. Lees and T. Kubota, "Inviscid hypersonic flow over blunt-nosed slender bodies," J. Aeronaut. Sci. 24, 195 (1957).

${ }^{5}$ N. C. Freeman, R. F. Cash, and D. Bedder, "An experimental investigation of asymptotic hypersonic flows," J. Fluid Mech. 18, 379 (1964).

${ }^{6} \mathrm{G}$. S. Beavers, "Shock-wave shapes on hypersonic axisymmetric powerlaw bodies," AIAA J. 7, 2038 (1969).

${ }^{7}$ H. G. Hornung, "Some aspect of hypersonic flow over power law bodies," J. Fluid Mech. 39, 143 (1969).

${ }^{8}$ T. F. O'Brien and M. J. Lewis, "Power law shapes for leading-edge blunting with minimal shock standoff," J. Spacecr. Rockets 36, 653 (1999).

${ }^{9}$ R. P. Starkey and M. J. Lewis, "Simple analytical model for parametric studies of hypersonic waveriders," J. Spacecr. Rockets 36, 516 (1999).

${ }^{10}$ R. P. Starkey and M. J. Lewis, "Analytical off-design lift-to-drag ratio analysis for hypersonic waveriders," J. Spacecr. Rockets 37, 516 (2000).

${ }^{11}$ W. F. N. Santos, "Direct simulation Monte Carlo of rarefied hypersonic flow on power law shaped leading edges," Ph.D. dissertation, Department of Aerospace Engineering, University of Maryland, College Park, MD (2001).

${ }^{12}$ W. F. N. Santos and M. J. Lewis, "Power law shaped leading edges in rarefied hypersonic flow," J. Spacecr. Rockets 39, 917 (2002).

${ }^{13}$ W. F. N. Santos and M. J. Lewis, "Angle of attack effect on rarefied hypersonic flow over power law shaped leading edges," in 23rd Interna- 
tional Symposium on Rarefied Gas Dynamics, Whistler, BC, Canada, 2002.

${ }^{14}$ W. F. N. Santos and M. J. Lewis, "Aerodynamic heating performance of power law leading edges in rarefied hypersonic flow," in 36th AIAA Thermophysics Conference, AIAA Pap. 2003-3894, Orlando, FL (2003).

${ }^{15}$ W. F. N. Santos and M. J. Lewis, "Effects of compressibility on rarefied hypersonic flow over power law leading edges," in 42nd AIAA Aerospace Sciences Meeting and Exhibit, AIAA Pap. 2004-1181, Reno, NV (2004).

${ }^{16}$ W. F. N. Santos and M. J. Lewis, "DSMC calculations of rarefied hypersonic flow over power law leading edges with incomplete surface accommodation," in 34th AIAA Fluid Dynamics Conference and Exhibit, AIAA Pap. 2004-2636, Portland, OR (2004).

${ }^{17}$ W. F. N. Santos and M. J. Lewis, "Calculation of shock wave structure over power law bodies in hypersonic flow," J. Spacecr. Rockets 42, 213 (2005).

${ }^{18}$ W. F. N. Santos and M. J. Lewis, "Aerothermodynamic performance analysis of hypersonic flow on power law leading edges," J. Spacecr. Rockets 42, 588 (2005); presented at the 41st AIAA Aerospace Sciences Meeting and Exhibit, AIAA Pap. 2003-1134, Reno, NV (2003).

${ }^{19}$ W. F. N. Santos, "Leading-edge bluntness effects on aerodynamic heating and drag of power law body in low-density hypersonic flow," J. Braz. Soc. Mech. Sci. 27, 236 (2005).

${ }^{20}$ I. D. Boyd and J. F. Padilla, "Simulation of sharp leading edge aerothermodynamics," in 12th AIAA International Space Planes and Hypersonic Systems and Technologies, AIAA Pap. 2003-7062, Norfolk, VA (2003).

${ }^{21}$ M. D. Van Dyke, "A study of hypersonic small-disturbance theory," NACA TN-3173 (1954)

${ }^{22}$ W. G. Vincenti and C. H. Kruger, Introduction to Physical Gas Dynamics (Krieger, Malabar, 1986).

${ }^{23}$ H. Grad, "The profile of a steady plane shock wave," Commun. Pure Appl. Math. 5, 257 (1952).

${ }^{24}$ D. Gilbarg and D. Paolucci, "The structure of shock waves in the continuum theory of fluids," Arch. Ration. Mech. Anal. 2, 617 (1953).

${ }^{25}$ J. L. Anderson, "Tethered aerothermodynamic research for hypersonic waveriders," in Proceedings of the 1st International Hypersonic Waverider Symposium, University of Maryland, College Park, MD (1990).

${ }^{26}$ J. L. Potter and J. K. Rockaway, "Aerodynamic optimization for hypersonic flight at very high altitudes," in Rarefied Gas Dynamics: Space Science and Engineering, edited by B. D. Shizgal and D. P. Weaver (AIAA, New York, 1994), Vol. 160, p. 296.

${ }^{27}$ D. F. G. Rault, "Aerodynamic characteristics of a hypersonic viscous optimized waverider at high altitude," J. Spacecr. Rockets 31, 719 (1994).

${ }^{28}$ R. E. Graves and B. M. Argrow, "Aerodynamic performance of an osculating-cones waverider at high altitudes," in 35th AIAA Thermophysics Conference, AIAA Pap. 2001-2960, Anaheim, CA (2001).

${ }^{29}$ A. I. Shvets, V. I. Voronin, I. M. Blankson, V. Khikine, and L. Thomas, "On waverider performance with hypersonic flight speed and high alti- tudes," in 43rd AIAA Aerospace Sciences Meeting and Exhibit, AIAA Pap. 2005-0512, Reno, NV (2005).

${ }^{30}$ G. A. Bird, Molecular Gas Dynamics and the Direct Simulation of Gas Flows (Oxford University Press, Oxford, 1994).

${ }^{31}$ A. L. Garcia and W. Wagner, "Time step truncation error in direct simulation Monte Carlo," Phys. Fluids 12, 2621 (2000).

${ }^{32}$ N. G. Hadjiconstantinou, "Analysis of discretization in the direct simulation Monte Carlo," Phys. Fluids 12, 2634 (2000).

${ }^{33}$ G. A. Bird, "Monte Carlo simulation in an engineering context," in Progress in Astronautics and Aeronautics: Rarefied Gas Dynamics, edited by S. S. Fisher (AIAA, New York, 1981), Vol. 74, part I, p. 239.

${ }^{34} \mathrm{G}$. A. Bird, "Perception of numerical method in rarefied gas dynamics," in Rarefied Gas Dynamics: Theoretical and Computational Techniques, edited by E. P. Muntz, D. P. Weaver, and D. H. Campbell (AIAA, New York, 1989), Vol. 118, p. 374.

${ }^{35}$ C. Borgnakke and P. S. Larsen, "Statistical collision model for Monte Carlo simulation of polyatomic gas mixture," J. Comput. Phys. 18, 405 (1975).

${ }^{36}$ F. J. Alexander, A. L. Garcia, and, B. J. Alder, "Cell size dependence of transport coefficient in stochastic particle algorithms," Phys. Fluids 10, 1540 (1998).

${ }^{37}$ F. J. Alexander, A. L. Garcia, and B. J. Alder, "Erratum: Cell size dependence of transport coefficient is stochastic particle algorithms," Phys. Fluids 12, 731 (2000).

${ }^{38}$ B. L. Haas and M. A. Fallavollita, "Flow resolution and domain influence in rarefied hypersonic blunt-body flows," J. Thermophys. Heat Transfer 8, 751 (1994).

${ }^{39}$ K. Guo and G.-S. Liaw, "A review: Boundary conditions for the DSMC method," in 35th AIAA Thermophysics Conference, AIAA Pap. 20012953, Anaheim, CA (2001).

${ }^{40}$ J. Lubonski, "Hypersonic plane Couette flow in rarefied gas," Arch. Mech. Stosow. 14, 553 (1962).

${ }^{41}$ G. A. Bird, "The structure of rarefied gas flows past simple aerodynamic shapes," J. Fluid Mech. 36, 571 (1969).

${ }^{42}$ W. F. N. Santos, "Gas-surface interaction effect on round leading edge aerothermodynamics,” Braz. J. Phys. 37, 337 (2007).

${ }^{43}$ T. F. O'Brien, "Analysis of power law shaped leading-edge for waveriders designed with shock attachment," M.S. thesis, Department of Aerospace Engineering, University of Maryland, College Park, MD (1998).

${ }^{44}$ I. E. Vas, S. M. Bogdonoff, and A. G. Hammitt, "An experimental investigation of the flow over simple two-dimensional and axial symmetric bodies at hypersonic speeds," Jet Propul. 28, 97 (1958).

${ }^{45}$ J. F. McCarthy and T. Kubota, "A study of wakes behind a circular cylinder at $M=5.7$," AIAA J. 2, 629 (1964).

${ }^{46}$ R. N. Zapata, J. Haas, and G. K. Mruk, "Low Reynolds number effects on hypersonic flow over a two-dimensional cylinder," Adv. Appl. Mech. 4, 1161 (1967). 